\title{
Estimation of serum and salivary matrix metalloproteinase levels in oral squamous cell carcinoma patients: a systematic review and meta-analysis
}

\author{
Farzad Rezaei ${ }^{1}$, Mohammad Moslem Imani², Pia Lopez-Jornet ${ }^{3}$, Masoud Sadeghi ${ }^{4,5}$ \\ ${ }^{1}$ Department of Oral and Maxillofacial Surgery, Kermanshah University of Medical Sciences, Kermanshah, Iran \\ 2Department of Orthodontics, Kermanshah University of Medical Sciences, Kermanshah, Iran \\ ${ }^{3}$ Facultad de Medicina y Odontologia Universidad de Murcia, Hospital Morales Meseguer, Clinica Odontologic Adv Marques Velez s/n, \\ Spain \\ ${ }^{4}$ Medical Biology Research Center, Kermanshah University of Medical Sciences, Kermanshah, Iran \\ ${ }^{5}$ Students Research Committee, Kermanshah University of Medical Sciences, Kermanshah, Iran
}

Adv Dermatol Allergol 2021; XXXVIII (1): 106-114 DOI: https://doi.org/10.5114/ada.2021.104285

\begin{abstract}
Introduction: Matrix metalloproteinases (MMPs) play a pivotal role in the cancer progression, invasion, and angiogenesis.

Aim: This meta-analysis was conducted to evaluate the difference between oral squamous cell carcinoma (OSCC) patients and healthy controls in the serum and salivary MMP levels.

Material and methods: Four databases - Web of Science, PubMed, Scopus, and Cochrane Library - were searched up to March 2019. The pooled standard mean difference (SMD) and 95\% confidence interval (CI) were obtained to explain the difference between the patients and controls in the salivary and serum MMP levels. Both Egger's and Begg's tests were considered as the significant publication bias.

Results: Thirteen case-control studies were included in the meta-analysis. Among the analyses of serum MMP levels, the serum MMP7 (SMD = 0.78; 95\% Cl: 0.15-1.41; $p=0.02$ ) and MMP9 (SMD = 1.18; 95\% Cl: 0.51-1.84; $p=0.0005)$ levels were significantly higher in the OSCC patients than in the controls. In addition, the analyses of salivary MMP levels showed that the MMP1 (SMD $=0.46 ; 95 \% \mathrm{Cl}: 0.22-0.70 ; p=0.0001)$ and MMP9 $(\mathrm{SMD}=0.66 ; 95 \% \mathrm{Cl}$ : $0.19-1.12 ; p=0.005)$ levels were significantly higher in the OSCC patients than in the controls.

Conclusions: The meta-analysis showed that the serum MMP7 and MPP9 levels as well as the salivary MMP1 and MPP9 levels were significantly higher in the OSCC patients than in the controls.
\end{abstract}

Key words: oral squamous cell carcinoma, matrix metalloproteinase, serum, saliva.

\section{Introduction}

Oral carcinoma is the sixth most common malignancy worldwide [1]. Out of all new cases of oral carcinoma reported worldwide in 2012, 2/3 of the tumours were reported in developing countries, and South and Southeast Asia and some countries in southern Europe had the highest incidence. This carcinoma causes almost 145,328 deaths annually worldwide [2]. Oral squamous cell carcinoma (OSCC) - the most prevalent oral carcinoma - is an important health problem in the world, and around 600,000 cases are diagnosed annually [3]. Therefore, the prevalence and clinical pattern of OSCC change considerably depending on the geographical location where it is diagnosed [2]. Unfortunately, despite the advanced methods in surgery, radiotherapy, and chemotherapy, the mortality of OSCC is high [4]. When oral carcinoma is determined early in stages I-II, the patients' survival increases from $60 \%$ to $80 \%$ [5]. A suppressed immune system is an established phenomenon in OSCC patients and can include changes in cytokines and the balance of immune cells [6]. Development of molecular biology has shown that matrix metalloproteinases (MMPs) play

Address for correspondence: Masoud Sadeghi, Medical Biology Research Center, Kermanshah University of Medical Sciences, Kermanshah, Iran, e-mail: sadeghi_mbrc@yahoo.com Received: 23.07.2019, accepted: 4.08.2019. 
a pivotal role in the cancer progression, invasion, and angiogenesis [7]. Also, they impact the OSCC progression either by directly changing the extracellular environment or indirectly via beginning vascular regression $[8,9]$. The MMP family includes diverse substrates [10]. They are a large family of zinc-dependent endopeptidase and are critical to the physiologic degradation of the extracellular matrix [11]. MMPs are made up of a prodomain, a catalytic domain, a hinge region, and a hemopexin domain that have six separated groups [12]. Therefore, the measurement of some MMPs in OSCC patients may be examined for the diagnosis and prognosis of this disease [13].

\section{Aim}

This meta-analysis was conducted to determine the difference between the OSCC patients and the healthy controls in the serum and salivary MMP levels.

\section{Material and methods}

\section{Study protocol}

The protocol of the present meta-analysis was followed based on PRISMA guidelines [14].

\section{Focused question}

Are serum and salivary MMP levels (intervention) related to OSCC (outcome) compared to controls (comparison) in people (population)?

\section{Search strategy and study selection}

The electronic databases of Scopus, Web of Science, PubMed, and Cochrane Library were systematically searched to identify the relevant articles up to March 2019 without language restriction. The search terms ("matrix metalloproteinase" OR "MMP") AND ("OSCC" OR "oral squamous cell carcinoma" OR "oral SCC" OR "oral cancer" OR "oral carcinoma") AND ("serum” OR "saliva" OR "salivary" OR "blood") were used in the databases to find the relevant articles. In addition, the citations in the systematic review/review articles on the topic were checked and reviewed.

Two authors (M.S. and F.R.) conducted the search and reviewed all articles retrieved from the search. In the first stage, duplicate articles were excluded. In the second stage, the titles and abstracts of the remaining articles were screened to exclude irrelevant articles. In the third stage, full texts of articles that met the eligibility criteria were assessed, among which some full texts were excluded with reasons. At last, the remaining full texts were included in the systematic review and then in the meta-analysis. The disagreements about the extracted data between both authors were resolved by a discussion.

\section{Eligibility criteria}

Inclusion criteria: 1) case-control studies reporting salivary/serum MMP levels and including OSCC patients with any stage and healthy controls and 2) diagnosis of OSCC histologically and/or clinically. Exclusion criteria: commentaries, letters to the editor, editorials, case reports, reviews/systematic reviews, conference abstracts, book chapters, and studies with irrelevant data.

\section{Data extraction}

Some data were retrieved from each study, including the first author's name, publication year, number of patients and controls, mean age and male/female ratio of patients and controls, measurement method of serum and salivary MMP levels, type of measured sample, and type of measured MMP. One author (M.S.) did data extraction and another author (F.R.) re-checked it.

\section{Quality assessment}

Critical evaluation of the included studies was organized using the quality assessment tool of the Newcastle-Ottawa Scale (NOS), with a maximum score of 9 for each study [15]. This tool was used to assess the risk of bias in individual studies and to grade the study quality as high ( $\geq 7)$, fair (4-7), or low $(<4)$. The quality assessment was conducted by one author (M.S.).

\section{Statistical analysis}

The standard mean difference (SMD) and its 95\% confidence interval $(\mathrm{Cl})$ were obtained for each study by Review Manager 5.3 (RevMan 5.3, Cochrane Collaboration) to explain the difference between the OSCC patients and the healthy controls in the salivary and serum MMP levels ( $p$-value less than 0.05 was considered statistically significant). The degree of heterogeneity was estimated using the $R^{2}$ statistic among the studies. $P<0.1(R>50 \%)$ showed a significant heterogeneity, following which the randomeffects model was conducted. The analysis of funnel plot was done by the Comprehensive Meta-Analysis version 2.0 (CMA 2.0) software using both Egger's and Begg's tests with a $p<0.05$ (2-tailed) considered to be a significant publication bias. We used the removal of one study and cumulative analysis to assess the stability/consistency of the pooled results. The unit of salivary and serum levels of MMPs was $\mathrm{ng} / \mathrm{ml}$ in the present meta-analysis. If there was median (interquartile range) in the studies, we converted it to mean \pm SD by formula [16].

\section{Results}

\section{Study selection}

Figure 1 shows the process of the literature search. Out of 221 studies retrieved from the databases. After removing duplicate and irrelevant studies, 21 studies 
were assessed based on their full texts. Out of 21 studies, 9 studies were excluded for reasons given ( 2 had no control group, 1 was a review, 1 was a book chapter, 2 had irrelevant data, 2 included laryngeal cancers, and 1 reported a polymorphism of MMP). After that, 13 studies were included in systematic review, from which 9 studies were reported on serum and 5 studies on salivary levels. The number of studies reporting MMP levels in serum or saliva is shown in Figure 1. At last, 13 studies (9 on serum and 5 on salivary levels) were analysed in the meta-analysis (serum MMPs 2, 3, 7, 9, and 13 and salivary MMPs 1, 3, 9).

\section{Study characteristics}

The characteristics of the included case-control studies are presented in Table 1. Out of thirteen studies included in the meta-analysis, five studies were from Iran [4, 17-20], two from China [6, 21], two from Germany [22, 23], one from Korea [24], one from Taiwan [25], one from Egypt [26], and one from Pakistan [27]. The studies had been published from 2008 to 2019. Four studies [4, 20, 22, 25] reported MMP levels on saliva and eight studies $[6,17-19,21,23,24,27]$ on serum and one study [26] included both samples. The measurement method of saliva or serum levels of MMPs was ELISA except for one study [25] that was mass spectrometry. Among all studies, nine studies included the OSCC patients with stages I to IV [6, 17-19, 21-25], two studies stages I and II [4, 26], and two studies $[20,27]$ did not report. The rest of data, including the number, mean age, and male/female ratio of OSCC patients and controls are shown in Table 1 . Two studies $[22,27]$ reported median (interquartile range), which was converted to mean \pm SD.

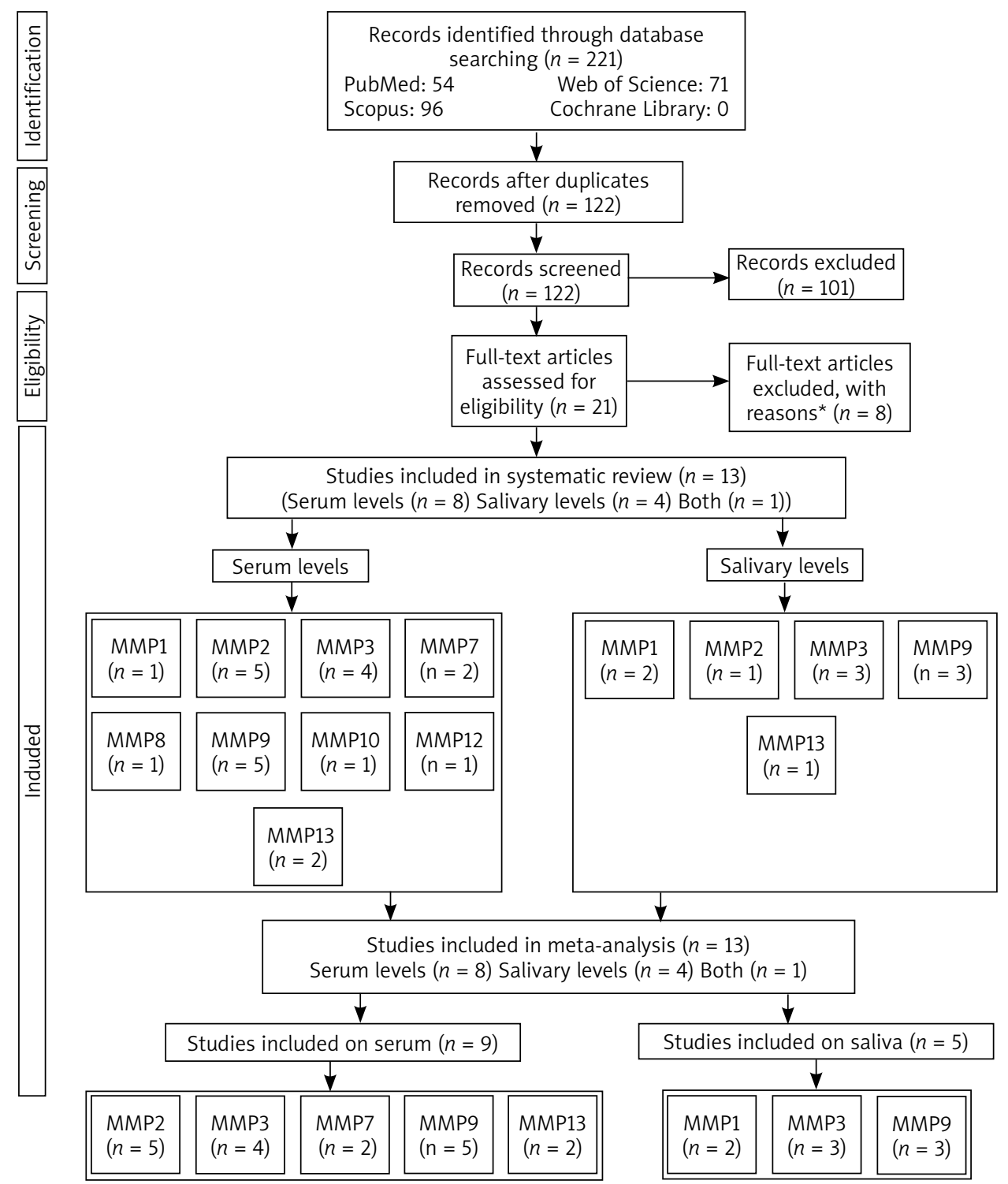

Figure 1. Flow chart of the present meta-analysis 
Table 1. Characteristics of included case-control studies

\begin{tabular}{|c|c|c|c|c|c|c|c|c|c|c|c|}
\hline $\begin{array}{l}\text { First author, } \\
\text { publication } \\
\text { year }\end{array}$ & Country & $\begin{array}{c}\text { No. of } \\
\text { patients }\end{array}$ & $\begin{array}{l}\text { No. of } \\
\text { controls }\end{array}$ & $\begin{array}{c}\text { Patients } \\
\text { (male/ } \\
\text { female) }\end{array}$ & $\begin{array}{c}\text { Controls } \\
\text { (male/ } \\
\text { female) }\end{array}$ & $\begin{array}{l}\text { Patients } \\
\text { (mean age) } \\
\text { [years] }\end{array}$ & $\begin{array}{l}\text { Controls } \\
\text { (mean age) } \\
\text { [years] }\end{array}$ & Method & Sample & $\begin{array}{l}\text { Measured } \\
\text { MMP }\end{array}$ & $\begin{array}{l}\text { Stage } \\
\text { of } \\
\text { OSCC }\end{array}$ \\
\hline $\begin{array}{l}\text { Lee, } 2008 \\
{[24]}\end{array}$ & Korea & 37 & 20 & NA & NA & 59.1 & NA & ELISA & Serum & MMP2, MMP9 & I-IV \\
\hline $\begin{array}{l}\text { Tadbir, } \\
2012 \text { [17] }\end{array}$ & Iran & 45 & 45 & $22 / 23$ & $22 / 23$ & 57.0 & 56.6 & ELISA & Serum & MMP3 & I-IV \\
\hline $\begin{array}{l}\text { Chang, } \\
2013[21]\end{array}$ & China & 151 & 111 & $142 / 9$ & $101 / 10$ & 52.5 & 48.3 & ELISA & Serum & MMP2, MMP9 & I-IV \\
\hline $\begin{array}{l}\text { Andisheh- } \\
\text { Tadbir, } \\
2014 \text { [18] }\end{array}$ & Iran & 45 & 45 & $22 / 23$ & $22 / 23$ & 57 & 56.6 & ELISA & Serum & MMP3 & I-IV \\
\hline $\begin{array}{l}\text { Lotfi, } 2015 \\
\text { [19] }\end{array}$ & Iran & 20 & 20 & $11 / 9$ & $10 / 10$ & 61.3 & 53.9 & ELISA & Serum & MMP2, MMP9 & I-IV \\
\hline $\begin{array}{l}\text { Jiang, } 2016 \\
\text { [6] }\end{array}$ & China & 204 & 212 & $157 / 47$ & $155 / 57$ & 57.3 & 56.6 & ELISA & Serum & MMP7 & I-IV \\
\hline $\begin{array}{l}\text { Yu, } 2016 \\
{[25]}\end{array}$ & Taiwan & 131 & 96 & $129 / 2$ & $96 / 0$ & 52.5 & 48.8 & $\begin{array}{l}\text { LC-MRM- } \\
\text { MS }\end{array}$ & Saliva & $\begin{array}{c}\text { MMP1, MMP3, } \\
\text { MMP9 }\end{array}$ & I-IV \\
\hline $\begin{array}{l}\text { Ghallab, } \\
2017 \text { [26] }\end{array}$ & Egypt & 15 & 15 & $6 / 9$ & $6 / 9$ & 47.7 & 43.3 & ELISA & $\begin{array}{l}\text { Serum, } \\
\text { saliva }\end{array}$ & MMP9 & I, II \\
\hline $\begin{array}{l}\text { Nosratzehi, } \\
2017 \text { [20] }\end{array}$ & Iran & 30 & 30 & NA & NA & NA & NA & ELISA & Saliva & $\begin{array}{l}\text { MMP1, MMP2 } \\
\text { MMP3, MMP13 }\end{array}$ & NA \\
\hline $\begin{array}{l}\text { Peisker, } \\
2017 \text { [22] }\end{array}$ & Germany & 30 & 30 & $14 / 16$ & $18 / 12$ & 65.0 & 60.7 & ELISA & Saliva & MMP9 & I-IV \\
\hline $\begin{array}{l}\text { Schiegnitz, } \\
2017 \text { [23] }\end{array}$ & Germany & 81 & 49 & NA & NA & 68.0 & 58.0 & ELISA & Serum & $\begin{array}{l}\text { MMP2, MMP3, } \\
\text { MMP13 }\end{array}$ & I-IV \\
\hline $\begin{array}{l}\text { Lee, } 2018 \\
{[24]}\end{array}$ & Iran & 15 & 30 & $9 / 6$ & NA & NA & NA & ELISA & Saliva & MMP3 & I, II \\
\hline $\begin{array}{l}\text { Choudhry, } \\
2019 \text { [27] }\end{array}$ & Pakistan & 38 & 38 & $26 / 12$ & $23 / 15$ & 50.9 & 50.8 & ELISA & Serum & $\begin{array}{c}\text { MMP1, MMP2 } \\
\text { MMP3, MMP7 } \\
\text { MMP8, MMP9 } \\
\text { MMP10, MP12, } \\
\text { MMP13 }\end{array}$ & NA \\
\hline
\end{tabular}

LC-MRM-MS - liquid chromatography multiple reaction monitoring mass spectrometry, MMP - matrix metalloproteinase, NA - not available.

\section{Meta-analysis reports \\ Serum levels}

Figure 2 shows serum levels of MMPs 2, 3, 7, 9, and 13 in OSCC and healthy controls. The serum MMP2 level was evaluated in five studies [19, 21, 23, 24, 27] with a total of 327 OSCC patients and 238 controls, the pooled SMD of which was found to be $0.49 \mathrm{ng} / \mathrm{ml}(95 \% \mathrm{Cl}:-0.47,1.45$; $\left.p=0.32 ; P^{2}=96 \%, P_{h}<0.00001\right)$. The serum MMP3 level of 209 patients and 177 controls in four studies [17, 18, $23,27]$ reached a pooled SMD of $0.44(95 \% \mathrm{Cl}:-0.03$, $\left.0.91 ; p=0.06 ; R^{2}=80 \%, P_{h}=0.002\right)$. The serum level of MMP7 was reported in two studies $[6,27]$ on 242 patients and 250 controls, the pooled SMD of which was 0.78 (95\% Cl: $\left.0.15,1.41 ; p=0.02 ; l^{2}=84 \%, P_{h}=0.01\right)$. A total of 261 patients and 204 controls were included in five studies $[19,21,24,26,27]$ for analysis of the serum level of MMP9 with a pooled SMD of $1.18(95 \% \mathrm{Cl}$ : $\left.0.51,1.84 ; p=0.0005 ; P^{2}=88 \%, P_{h}<0.00001\right)$. In addition,
119 patients and 87 controls were included in two studies $[23,27]$ showing the serum MMP13 level, the pooled SMD of which was obtained to be 0.42 ( $95 \% \mathrm{Cl}$ : $-0.05,0.90$; $\left.p=0.08 ; P=63 \%, P_{h}=0.10\right)$. Therefore, among the analyses of serum MMP levels, just the serum MMPs 7 and 9 levels were significantly higher in the OSCC patients than the healthy controls.

\section{Salivary levels}

Figure 3 illustrates the pooled analysis of the salivary levels of MMPs in the OSCC patients compared to the healthy controls. Two studies $[25,26]$ including a total of 161 patients and 126 controls reported that the pooled SMD of the salivary MMP1 level was 0.46 (95\% Cl: 0.22 , $\left.0.70 ; p=0.0001 ; R^{2}=0.43 \%, P_{h}=0.19\right)$. Three studies [4, $20,25]$ included a total of 176 patients and 156 controls and reported the pooled SMD of 0.45 (95\% Cl: 0.00, 0.91; $\left.p=0.05 ; P^{2}=66 \%, P_{h}=0.05\right)$. In addition, three studies in- 


\section{MMP2}

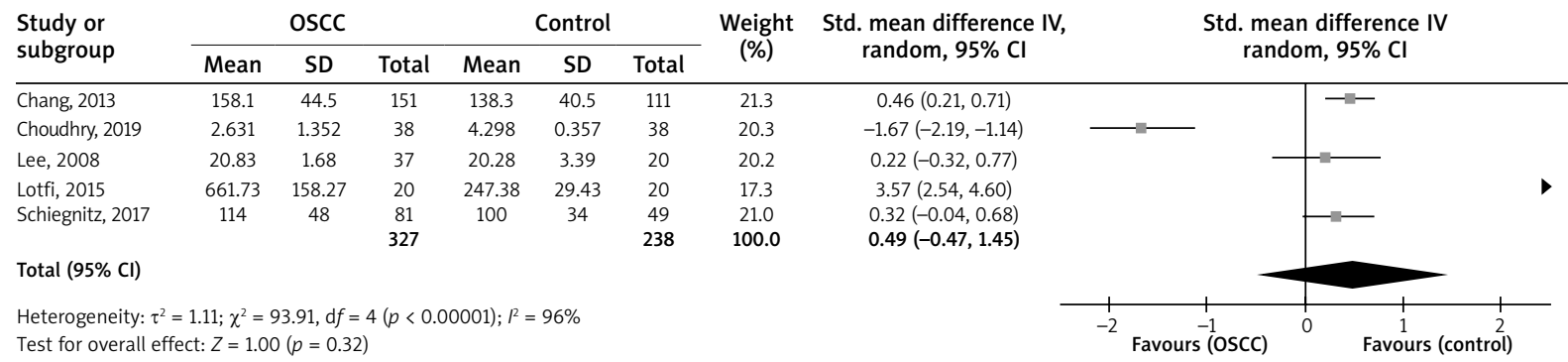

\section{MMP3}

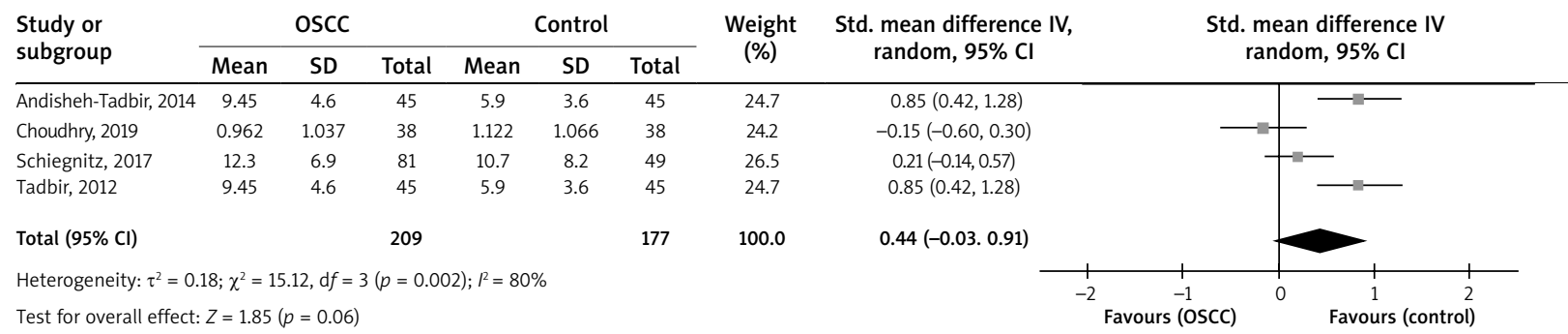

3. MMP7

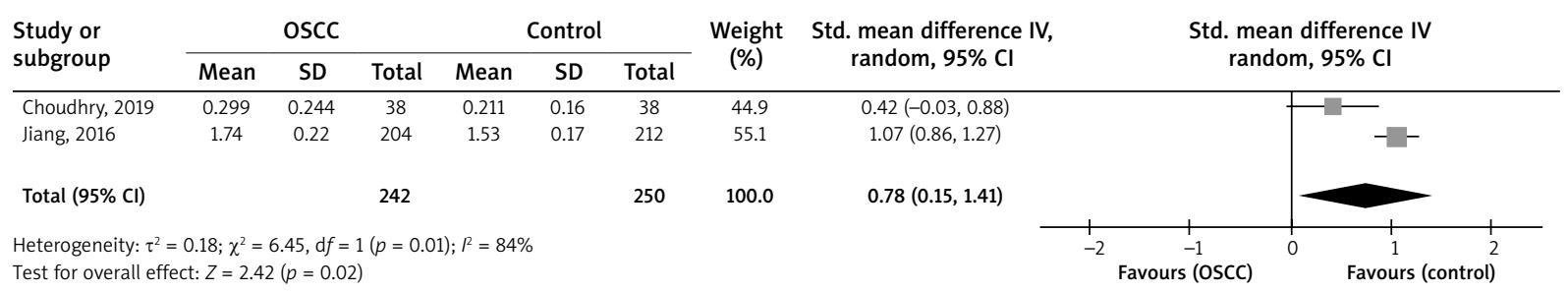

\section{MMP9}

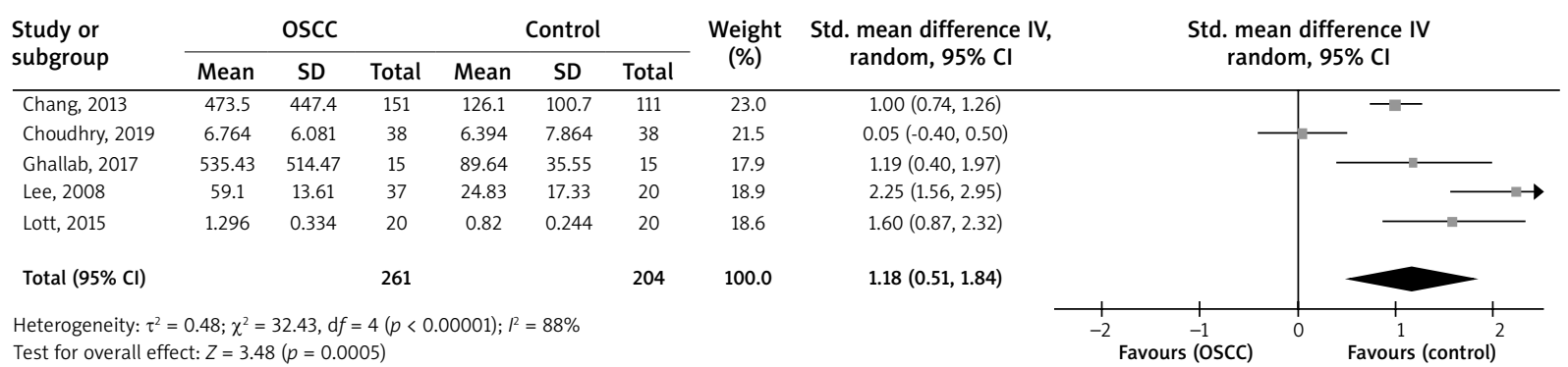

\section{MMP13}

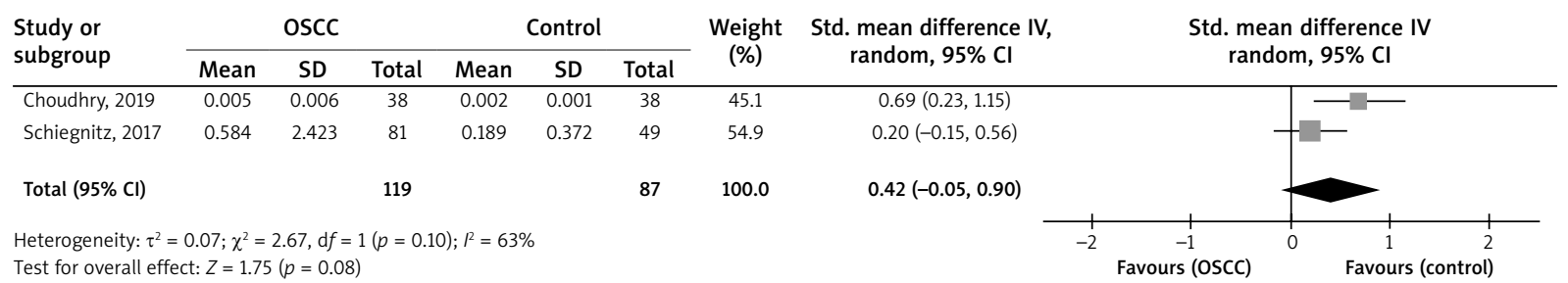

MMP-matrix metalloproteinase.

Figure 2. Analysis of the standard mean difference of the serum MMP levels in the oral squamous cell carcinoma (OSCC) patients compared to the healthy controls 
1. MMP1

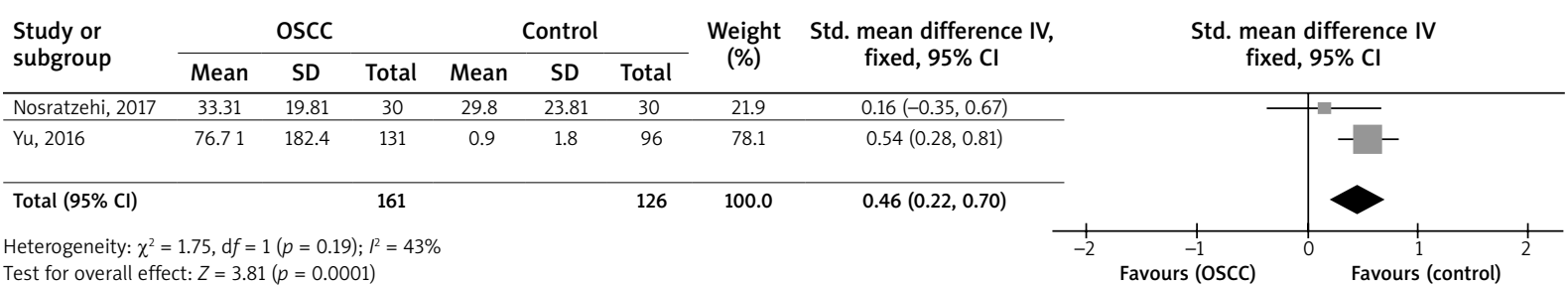

\section{MMP3}

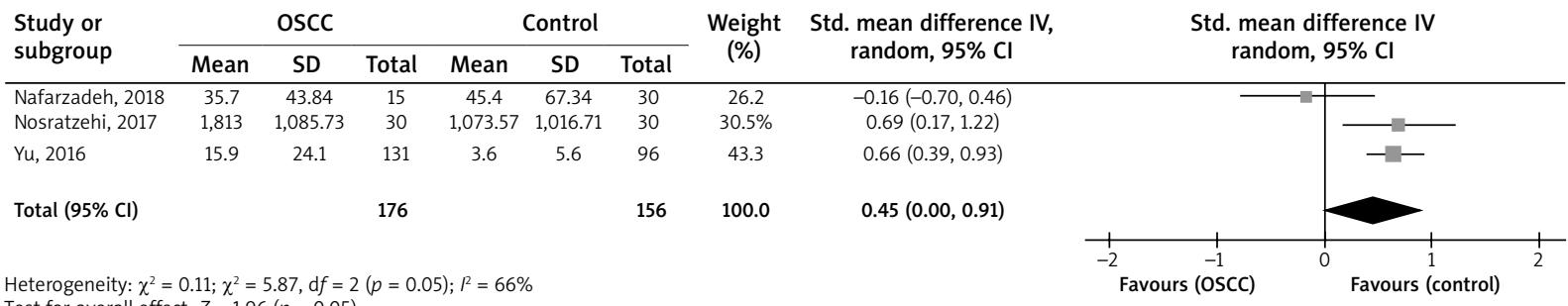

Test for overall effect: $Z=1.96(p=0.05)$

3. MMP9

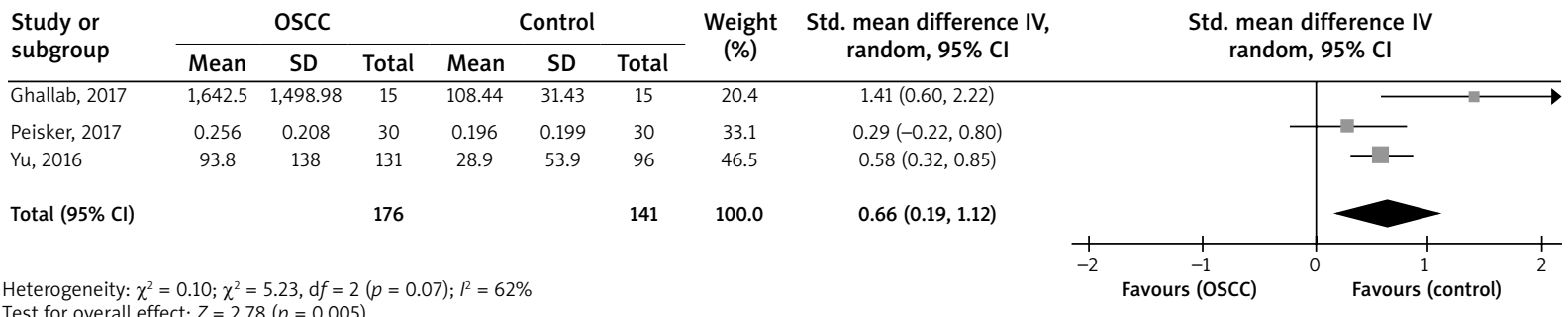

Test for overall effect: $Z=2.78(p=0.005)$

MMP - matrix metalloproteinase.

Figure 3. Analysis of the standard mean difference of the salivary MMP levels in the oral squamous cell carcinoma (OSCC) patients compared to the healthy controls

Table 2. Quality assessment of the included case-control studies

\begin{tabular}{|c|c|c|c|c|}
\hline First author, publication year & Selection & Comparability\# & Exposure & Total points \\
\hline Lee, 2008 [24] & $* *$ & - & $* * *$ & 5 \\
\hline Tadbir, 2012 [17] & $* * * *$ & ** & $* * *$ & 9 \\
\hline Chang, 2013 [21] & $* * *$ & ** & $* * *$ & 8 \\
\hline Andisheh-Tadbir, 2014 [18] & $* * * *$ & $* *$ & $* * *$ & 9 \\
\hline Lotfi, 2015 [19] & $* * *$ & ** & $* * *$ & 8 \\
\hline Jiang, 2016 [6] & $* * *$ & ** & $* * *$ & 8 \\
\hline Yu, 2016 [25] & ** & * & $* * *$ & 6 \\
\hline Ghallab, 2017 [26] & $\star * \star * \star$ & ** & 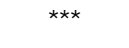 & 9 \\
\hline Nosratzehi, 2017 [20] & ** & - & $* * *$ & 5 \\
\hline Peisker, 2017 [22] & $* * *$ & ** & $* * *$ & 8 \\
\hline Schiegnitz, 2017 [23] & $* *$ & * & $* * *$ & 6 \\
\hline Lee, 2018 [24] & $* * *$ & - & $* \star \star$ & 6 \\
\hline Choudhry, 2019 [27] & $* * *$ & ** & $* * *$ & 8 \\
\hline
\end{tabular}

\#Two groups matched for age (one star) and sex (one star). 

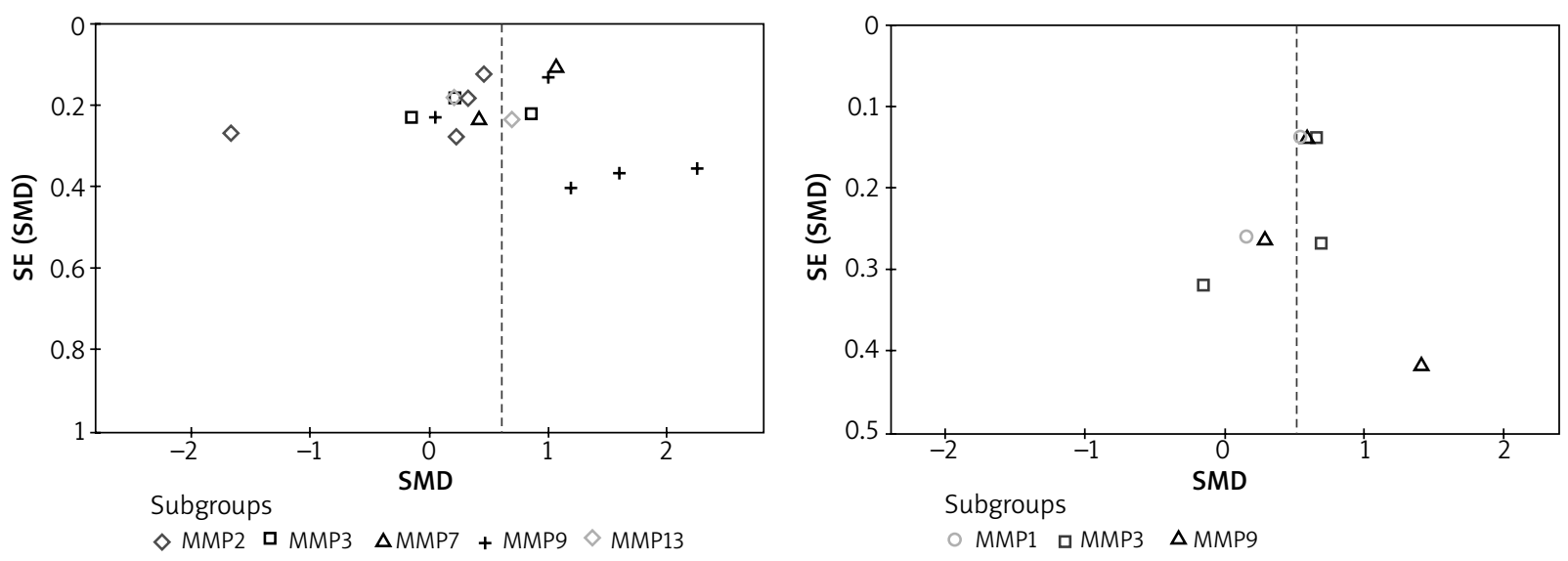

Figure 4. Funnel plot of the standard mean difference (SMD) of the serum and salivary MMP levels in the oral squamous cell carcinoma patients compared to the healthy controls

cluded a total of 176 patients and 141 controls [22, 25, 26] and showed that the pooled SMD for the salivary MMP9 level was $0.66\left(95 \% \mathrm{Cl}: 0.19,1.12 ; p=0.005 ;{ }^{2}=62 \%\right.$, $\left.P_{h}=0.07\right)$. Therefore, analyses of the salivary levels showed that the MMPs 1 and 9 levels were significantly higher in the OSCC patients than the controls.

\section{Quality assessment}

The results of quality assessment showed that the mean square of all included studies was 7.3 (Table 2). Eight studies had high quality (score $\geq 7$ ) and five studies had fair quality (score of 4-7).

\section{Sensitivity analysis}

We used two sensitivity analyses (removal of one study and cumulative analysis) on the pooled analyses with a minimum of three studies included in the analysis. Both sensitivity analyses did not change the previous results and therefore showed the stability of the previous results.

\section{Publication bias}

Both Egger's and Begg's tests were used for the analyses with a minimum of three studies included in the analysis. Both tests did not reveal any publication bias across the studies in all the analyses (Figure 4).

\section{Discussion}

The present meta-analysis evaluated the serum MMPs 2, 3, 7, 9, and 13 levels and the salivary MMPs 1, 3 , and 9 levels. The results showed the serum MMPs 7 and 9 levels and also the salivary MMPs 1 and 9 levels were significantly higher in the OSCC patients than the controls. With regard to the salivary levels of MMPs in the OSCC patients compared to the controls, out of five stud- ies $[19,21,23,24,27]$ reporting the serum MMP2 level, two studies [19, 21] showed significantly elevated levels and one study [13] showed a significantly decreased level. Four studies $[17,18,23,27]$ reported the serum MMP3 level, from which two studies $[18,23]$ reported a significantly elevated level. The serum MMP7 level was reported in two studies [6, 27], of which one study [6] indicated a significantly elevated level. Out of five studies [19, 21, $24,26,27]$ reporting the serum MMP9 level, four studies $[19,21,24,26]$ showed significantly elevated levels. The serum MMP13 level was reported in two studies [23, 27], of which one study [13] showed a significantly elevated level. With regard to the salivary levels of MMPs in the OSCC patients compared to the controls, two $[25,26]$, three $[4,25,26]$, and three studies $[22,25,26]$ reported MMPs 1, 3, and 9 levels, respectively. The results showed that one [25], two [20, 25], and two studies [25, 26] showed significantly elevated levels.

One study [18] found no correlation between the serum MMP3 level and clinicopathological characteristics of the OSCC tumour, such as lymph node metastasis, size, grade, and stage; whereas, some previous studies have obtained a connection between MMP3 expression and clinicopathological characteristics of OSCC $[28,29]$ as well as levels of MMPs 1 and 10 [30]. Kurahara et al. [28] observed a direct correlation between MMP3 expression and incidence of lymph node metastasis. MMP13 is associated with the invasion depth and poor prognosis of OSCC [31]. A review study [32] showed that upregulation of MMPs 1 and 9 could be used as markers for malignant transformation to oral cancer. Studies have demonstrated that MMP13 is produced directly by the cancer tissue and indirectly advances the tumour angiogenesis $[33,34]$. Baker et al. [35] proved that MMP3 expression was predominantly higher in OSCC than in normal tissue. A meta-analysis [36] on the Asian and European popu- 
lations showed no significant connection between the MMP3 level and the risk of head and neck cancer, but in the European subgroup a MMP3 polymorphism was significantly related to the risk of head and neck cancer. Some reports have suggested that MMP9 expression may be an effective indicator for predicting the early stage of OSCC and also a good aim for therapeutic intervention [37, 38].

Vascular endothelial growth factor (VEGF) can increase MMP expression in endothelial cells [39]. A significant correlation has been found between serum levels of VEGF and MMP3 in the OSCC patients [18]. MMP9 may act as a proangiogenic indicator during tumorigenesis by raising the VEGF activity [40, 41]. Previous research has shown that tumour necrosis factor $\alpha$ and transforming growth factor $\beta 1$ increase MMPs 2 and 9 RNA and protein expression levels in the lesion sites of OSCC [42, 43]. Zhang et al. [44] reported elevated levels of MMP1 in OSCC correlated fibroblasts compared with normal fibroblasts. Chemerin has been presented to stimulate MMP9 and other MMPs in human endothelial cells and chondrocytes, respectively $[45,46]$. Thrailkill et al. [47] proposed a direct correlation between the serum MMP3 level and age. Also, one research [17] showed that the serum MMP3 level was higher in females than in males. Another research found different MMP3 serum levels in males and females [48]. These results show that age, sex, and other markers can affect MMPs levels and the pathological conditions of OSCC.

With regard to distinguishing OSCC from controls, the area under curve (AUC) rates obtained were 0.651 and 0.886 for serum MMPs 2 and 9, respectively [21]. Choudhry et al. [27] showed that the serum levels of MMPs 1, 2, 3, 7, 8, 9, 10, 12, and 13 had an AUC higher than 0.500. Another study [26] found an AUC of 1.00 for salivary and serum MMP9. Peisker et al. [22] reported an AUC of 0.698 for salivary MMP9. In addition, AUC was more than 0.500 for serum MMPs 2, 3, and 13 [23]. The results of AUC show that the MMP levels can distinguish OSCC from controls, which can be stronger or better than others based on some MMPs. Future studies are recommended to pay more attention to this point that MMPs can make higher differentiation.

Limitations: 1) Measurement of MMP levels was the second outcome in some studies. 2) Due to few studies reported, we could not conduct subgroup analysis. 3) Some studies had different treatments (chemotherapy, surgery, and radiotherapy) for patients and some studies had no treatment. 4) Different percentages were found for OSCC stages among the studies. 5) A number of studies included smokers and alcohol consumers as inclusion criteria. 6) There was high heterogeneity in most analyses. Strengths: 1) There was no publication bias. 2) The stability of the results was confirmed. 3) More than 60\% of studies had high quality. 4) Most studies had a similar method for measurement of MMP levels. 5) In most studies, there were age- and/or sex-matched controls.

\section{Conclusions}

Considering the limitations and few studies reported, in particular, this meta-analysis showed that the serum MMPs 7 and 9 levels and the salivary MMPs 1 and 9 levels were significantly higher in the OSCC patients than in controls. However, due to high heterogeneity among the studies analysed in the meta-analysis, readers should construe the results more carefully and precisely. Future studies should be done with a larger sample size in different areas to confirm the usefulness of these MMPs in tumour detection or progression.

\section{Acknowledgments}

We sincerely and gratefully thank the Kermanshah University of Medical Sciences for providing the financial support (project code: 980754).

\section{Conflict of interest}

The authors declare no conflict of interest.

\section{References}

1. Warnakulasuriya S. Causes of oral cancer - an appraisal of controversies. Br Dent J 2009; 207: 471-5.

2. Ghantous Y, Abu Elnaaj I. Global incidence and risk factors of oral cancer. Harefuah 2017; 156: 645-9.

3. Parkin DM, Bray F, Ferlay J, Pisani P. Global cancer statistics, 2002. CA Cancer J Clin 2005; 55: 74-108.

4. Nafarzadeh S, Emamgholipour M, Bijani F, et al. Is salivary evaluation of P53 and MMP-3 a good tool for early detection of oral squamous cell carcinoma? J Contemp Med Sci 2018; 4: 176-78.

5. Mehrotra R, Gupta DK. Exciting new advances in oral cancer diagnosis: avenues to early detection. Head Neck Oncol 2011; 3: 33.

6. Jiang T, Xie P, Liu H. Circulating anti-matrix metalloproteinase-7 antibodies may be a potential biomarker for oral squamous cell carcinoma. J Oral Maxillofac Surg 2016; 74: 650-7.

7. Kessenbrock K, Plaks V, Werb Z. Matrix metalloproteinases: regulators of the tumor microenvironment. Cell 2010; 141: 52-67.

8. Brown GT, Murray GI. Current mechanistic insights into the roles of matrix metalloproteinases in tumour invasion and metastasis. J Pathol 2015; 237: 273-81.

9. Da Silva SD, Morand GB, Alobaid FA, et al. Epithelial-mesenchymal transition (EMT) markers have prognostic impact in multiple primary oral squamous cell carcinoma. Clin Exp Metastasis 2015; 32: 55-63.

10. Egeblad M, Werb Z. New functions for the matrix metalloproteinases in cancer progression. Nat Rev Cancer 2002; 2: 161-74.

11. Chen Y, Zhang W, Geng N, et al. MMPs, TIMP-2, and TGF-b1 in the cancerization of oral lichen planus. Head Neck 2008; 30: 1237-45.

12. Visse R, Nagase H. Matrix metalloproteinases and tissue inhibitors of metalloproteinases: structure, function, and biochemistry. Circ Res 2003; 92: 827-39.

13. Mishev G, Deliverska E, Hlushchuk R, et al. Prognostic value of matrix metalloproteinases in oral squamous cell carcinoma. Biotechnol Biotechnol Equip 2014; 28: 1138-49.

14. Moher D, Liberati A, Tetzlaff J, et al. Preferred reporting items for systematic reviews and meta-analyses: the PRISMA statement. PLoS Med 2009; 6: e1000097. 
15. Wells GA, Shea B, O'Connell D, et al. The Newcastle-Ottawa scale (NOS) for assessing the quality of non-randomised studies in meta-analyses. Ottawa: Ottawa Hospital Research Institute; 2011. http://www.ohri.ca/programs/clinical_epidemiology/oxford.asp. Accessed 12 Jan 2016.

16. Wan X, Wang W, Liu J, Tong T. Estimating the sample mean and standard deviation from the sample size, median, range and/ or interquartile range. BMC Med Res Methodol 2014; 14: 135.

17. Tadbir AA, Purshahidi S, Ebrahimi H, et al. Serum level of MMP3 in patients with oral squamous cell carcinoma: lack of as sociation with clinico-pathological features. Asian Pac J Cancer Prev 2012; 13: 4545-8.

18. Andisheh-Tadbir A, Khademi B, Kamali F, et al. Upregulation of serum vascular endothelial growth factor and matrix metalloproteinase-3 in patients with oral squamous cell carcinoma. Tumour Biol 2014; 35: 5689-93.

19. Lotfi A, Mohammadi G, Tavassoli A, et al. Serum levels of MMP9 and MMP2 in patients with oral squamous cell carcinoma. Asian Pac J Cancer Prev 2015; 16: 1327-30.

20. Nosratzehi T, Alijani E, Moodi M. Salivary MMP-1, MMP-2, MMP3 and MMP-13 levels in patients with oral lichen planus and squamous cell carcinoma. Asian Pac J Cancer Prev 2017; 18 : 1947-51.

21. Chang PY, Kuo YB, Wu TL, et al. Association and prognostic value of serum inflammation markers in patients with leukoplakia and oral cavity cancer. Clin Chem Lab Med 2013; 51: 1291-300.

22. Peisker A, Raschke GF, Fahmy MD, et al. Salivary MMP-9 in the detection of oral squamous cell carcinoma. Med Oral Patol Oral Cir Bucal 2017; 22: e270-5.

23. Schiegnitz E, Kämmerer PW, Schön H, et al. The matrix metalloproteinase and insulin-like growth factor system in oral cancer - a prospective clinical study. Onco Targets Ther 2017; 10: 5099-105.

24. Lee BK, Kim MJ, Jang HS, et al. A high concentration of MMP-2/ gelatinase $A$ and MMP-9/gelatinase $B$ reduce NK cell-mediated cytotoxicity against an oral squamous cell carcinoma cell line. In Vivo 2008; 22: 593-7.

25. Yu JS, Chen YT, Chiang WF, et al. Saliva protein biomarkers to detect oral squamous cell carcinoma in a high-risk population in Taiwan. Proc Natl Acad Sci USA 2016; 113: 11549-54.

26. Ghallab NA, Shaker OG. Serum and salivary levels of chemerin and MMP-9 in oral squamous cell carcinoma and oral premalignant lesions. Clin Oral Investig 2017; 21: 937-47.

27. Choudhry N, Sarmad S, Waheed NUA, Gondal AJ. Estimation of serum matrix metalloproteinases among patients of oral squamous cell carcinoma. Pak J Med Sci 2019; 35: 252-6.

28. Kurahara S, Shinohara M, Ikebe T, et al. Expression of MMPS MT-MMP, and TIMPs in squamous cell carcinoma of the ora cavity: correlations with tumor invasion and metastasis. Head Neck 1999; 21: 627-38.

29. Kusukawa J, Sasaguri Y, Morimatsu M, Kameyama T. Expression of matrix metalloproteinase-3 in stage I and II squamous cel carcinoma of the oral cavity. J Oral Maxillofac Surg Off J Am Assoc Oral Maxillofac Surg 1995; 53: 530-4.

30. $\mathrm{Ha} \mathrm{NH}, \mathrm{Woo} \mathrm{BH}, \mathrm{Ha}$ ES, et al. Prolonged and repetitive exposure to Porphyromonas gingivalis increases aggressiveness of ora cancer cells by promoting acquisition of cancer stem cell properties. Tumour Biol 2015; 36: 9947-60.

31. Mäkinen LK, Häyry V, Atula T, et al. Prognostic significance of matrix metalloproteinase-2,-8,-9, and-13 in oral tongue cancer. J Oral Pathol Med 2012; 41: 394-9.

32. Jordan RC, Macabeo-Ong M, Shiboski CH, et al. Overexpression of matrix metalloproteinase-1 and -9 mRNA is associated with progression of oral dysplasia to cancer. Clin Cancer Res 2004; 61: 6460-5.

33. Kudo Y, lizuka S, Yoshida M, et al. Matrix metalloproteinase-13 (MMP-13) directly and indirectly promotes tumor angiogenesis. J Biol Chem 2012; 287: 38716-28.

34. Lederle W, Hartenstein B, Meides A, et al. MMP13 as a stromal mediator in controlling persistent angiogenesis in skin carcinoma. Carcinogenesis 2010; 31: 1175-84.

35. Baker EA, Leaper DJ, Hayter JP, Dickenson AJ. The matrix metalloproteinase system in oral squamous cell carcinoma. J Oral Maxillofac Surg 2006; 44: 482-6.

36. Zhang C, Li C, Zhu M, et al. Meta-analysis of MMP2, MMP3, and MMP9 promoter polymorphisms and head and neck cancer risk. PLoS One 2013; 8: e62023.

37. Katayama A, Bandoh N, Kishibe K, et al. Expressions of matrix metalloproteinases in early-stage oral squamous cell carcinoma as predictive indicators for tumor metastases and prognosis. Clin Cancer Res 2004; 10: 634-40.

38. de Vicente JC, Fresno MF, Villalain L, et al. Expression and clinical significance ofmatrixmetalloproteinase-2 and matrix metalloproteinase-9 in oral squamous cell carcinoma. Oral Oncol 2005; 41: 283-93.

39. Tas F, Duranyildiz D, Oguz H, et al. Circulating levels of vascular endothelial growth factor (VEGF), matrix metalloproteinase-3 (MMP-3), and BCL-2 in malignant melanoma. Med Oncol 2008; 25: 431-6.

40. Henriques AC, de Matos FR, Galvao HC, Freitas Rde A. Immunohistochemical expression of MMP-9 and VEGF in squamous cell carcinoma of the tongue. J Oral Sci 2012; 54: 105-11.

41. de Carvalho Fraga CA, Farias LC, de Oliveira MV, et al. Increased VEGFR2 and MMP9 protein levels are associated with epithelial dysplasia grading. Pathol Res Pract 2014; 210: 959-64.

42. Tortorici S, Mauro A, Burruano F, et al. Matrix metalloproteinase-2 matrix metalloproteinase- 9 and inducible nitric oxide synthase in oral leukoplakia: immunohistochemistry and RTPCR analysis. J Biol Regul Homeost Agents 2008; 22: 125-30.

43. Kato K, Hara A, Kuno T, et al. Matrix metalloproteinases 2 and 9 in oral squamous cell carcinomas: manifestation and localization of their activity. J Cancer Res Clin Oncol 2005; 131: 340-6.

44. Zhang Z, Tao D, Zhang P, et al. Hyaluronan synthase 2 expressed by cancer-associated fibroblasts promotes oral cancer invasion. J Exp Clin Cancer Res 2016; 35: 181.

45. Kaur J, Adya R, Tan BK, et al. Identification of chemerin receptor (ChemR23) in human endothelial cells: chemerin-induced endothelial angiogenesis. Biochem Biophys Res Commun 2010; 391: 1762-8

46. Berg V, Sveinbjornsson B, Bendiksen S, et al. Human articular chondrocytes express ChemR23 and chemerin; ChemR23 promotes inflammatory signalling upon binding the ligand chemerin(21-157). Arthritis Res Ther 2010; 12: R228.

47. Thrailkill KM, Moreau CS, Cockrell G, et al. Physiological matrix metalloproteinase concentrations in serum during childhood and adolescence, using Luminex Multiplex technology. Clin Chem Lab Med 2005; 43: 1392-9.

48. Komosinska-Vassev K, Olczyk P, Winsz-Szczotka K, et al. Ageand gender-dependent changes in connective tissue remodeling: physiological differences in circulating MMP-3, MMP-10, TIMP-1 and TIMP-2 level. Gerontology 2011; 57: 44-52. 